\title{
MicroRNA-181b expression in prostate cancer tissues and its influence on the biological behavior of the prostate cancer cell line PC-3
}

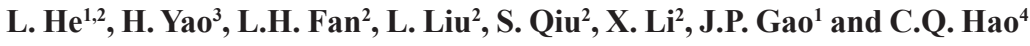 \\ ${ }^{1}$ Department of Urology, Chinese PLA General Hospital, Beijing, China \\ ${ }^{2}$ Department of Urology, Shenyang Military General Hospital, \\ Shenyang, China \\ ${ }^{3}$ Laboratory of Department of Gastroenterology, \\ Shenyang Military General Hospital, Shenyang, China \\ ${ }^{4}$ Department of Infectious Diseases, Tangdu Hospital, \\ Fourth Military Medical University, Xi'an, China \\ Corresponding authors: J.P. Gao / C.Q. Hao \\ E-mail: jiangpinggaocn@163.com / haochq@yahoo.com.cn
}

Genet. Mol. Res. 12 (2): 1012-1021 (2013)

Received June 21, 2012

Accepted September 5, 2012

Published April 2, 2013

DOI http://dx.doi.org/10.4238/2013.April.2.17

\begin{abstract}
We examined microRNA-181b (miRNA) expression in prostate cancer tissues and its effect on the prostate cancer cell line PC-3. Tissues from 27 cases of prostate cancer and 30 samples of normal human prostate were collected by surgical removal. Total miRNA was extracted, and the relative expression of miR-181b was quantified using RT-PCR. miR-181b ASO was transfected into prostate cancer PC-3 cells. miR-181b expression in transfected and non-transfected cells was measured using RT-PCR. Changes in cell apoptosis were measured using flow cytometry. MTT and cell growth curve methods were used to assess the influence of miR-181b expression on cell proliferation. The changes in cell invasive ability in vitro were detected using the Transwell chamber method. miR-181b was up-regulated in the prostate cancer tissues compared with the normal prostate samples. It was down-regulated after miR-181b ASO
\end{abstract}


transfection into the prostate cancer PC-3 cells. Down-regulation of $\mathrm{miR}-181 \mathrm{~b}$ in the PC-3 cell induced apoptosis, inhibited proliferation, and depressed invasion of $\mathrm{PC}-3$ cells in vitro. As miR-181b is overexpressed in prostate cancer, its down-regulation could have potential as gene therapy for prostate cancer by inducing apoptosis, inhibiting proliferation and depressing invasion by cancer cells.

Key words: miR-181b; Prostate cancer; PC-3; Apoptosis; Proliferation; Invasion

\section{INTRODUCTION}

Prostate cancer is one of the most common tumors among men in Europe and North America. In North America, mortality related to prostate cancer has surpassed that of lung cancer, ranking first among tumors that threaten male health (Jonler and Pedersen, 2007). In China, the incidence of prostate cancer has increased in recent years. The genesis of prostate cancer is associated with disequilibrium between the proto-oncogene and the anti-oncogene, which leads to uncontrolled proliferation of tumor cells (Gibson et al., 2007). Prostate cancer does not have specific symptoms at an early stage. Therefore, most patients have lost the opportunity for radical resection by the time the disease is detected.

Currently, androgen blocking is a commonly used treatment in which the clinical development of prostate cancer is controlled by reducing androgen in vivo. However, this method cannot achieve a radical effect. Approximately one-third of patients who receive androgen-blocking treatment experience a recurrence of prostate cancer, often a non-androgen-dependent refractory prostate cancer, within 18 months of treatment. Prostate cancer has a high mortality because effective treatments are still lacking. Malignant tumors can be radically cured only with knowledge of the mechanism of their genesis and development.

Biotherapy is currently the fourth treatment option after surgery, chemotherapy, and radiotherapy. It includes immunotherapy and gene therapy. Immunotherapy for prostate cancer is in both the preclinical study stage and phase I and II clinical trials. Gene therapy for malignant cancers is a prospective new method that has been developed in recent years. The application of gene therapy for prostate cancer has entered phase II clinical trials. Biotherapy is the most promising protocol for breakthroughs in androgen non-independent prostate cancer treatment (Xu and Wang, 2007).

miRNA is a type of noncoding single-stranded small RNA with a length of 18-25 oligonucleotides. miRNA completely or incompletely pairs with messenger RNA through its 3'-untranslated region to inhibit or disassociate posttranscriptional translation, which enables miRNA to participate in biological processes such as cell apoptosis, proliferation, differentiation, and development (Meltzer, 2005; Stark et al., 2005). miRNA expression is abnormal in prostate cancer tissues (Porkka et al., 2007; Ozen et al., 2008; Prueitt et al., 2008). The miRNA-181 family includes miRNA-181a, miRNA-181b, and miRNA-181c. They are prolific in human cells, and their expression is normal in many types of tumors (Nakajima et al., 2006; Debernardi et al., 2007; Marcucci et al., 2008; Shi et al., 2008; Chen et al., 2009). They play important roles in tumor genesis and development (Berezikov et al., 2005).

The current study explored the expression of miRNA-181b in prostate cancer tis- 
sues and the influence of miRNA-181b on the proliferation, apoptosis, and invasion of the prostate cancer cell line PC-3 to provide new insights and an experimental basis for prostate cancer treatment.

\section{MATERIAL AND METHODS}

\section{RNA extraction}

All prostate cancer tissue and normal tissue samples were obtained at the General Hospital of Shenyang Military Region. All samples were independently diagnosed by two pathologists. Total RNA was extracted from 27 prostate cancer tissues and 30 normal tissues. A portion of the frozen tissue was ground. Trizol (1000 $\mu \mathrm{L}$, Invitrogen, USA, Cat. No. 15596026) was added to $100 \mathrm{mg}$ tissue sample. After blowing with a sample injector, the tissue sample was transferred to a $1.5-\mathrm{mL}$ Eppendorf tube and cultured at room temperature for $5 \mathrm{~min}$. Chloroform $(200 \mu \mathrm{L})$ was added, oscillated, and centrifuged at $14,000 \mathrm{rpm}$ at $4^{\circ} \mathrm{C}$ for $15 \mathrm{~min}$. The supernatant was removed and transferred to an Eppendorf tube. Pre-chilled $\left(-20^{\circ} \mathrm{C}\right)$ isopropanol $(600 \mu \mathrm{L})$ was added, cultured at room temperature for $30 \mathrm{~min}$, and then centrifuged at 14,000 $\mathrm{rpm}$ at $4^{\circ} \mathrm{C}$ for $10 \mathrm{~min}$. The supernatant was discarded. Then, $1 \mathrm{~mL} 75 \%$ ethanol was added for precipitation pop-up. The solution was centrifuged at $12,000 \mathrm{rpm}$ for $10 \mathrm{~min}$. The supernatant was discarded. The sample was dried at room temperature for $5 \mathrm{~min}$. Then, $20 \mu \mathrm{L}$ RNase-free water was added for a 5 -min dissolution, and a $1-\mu \mathrm{L}$ sample solution was taken and dissolved in $9 \mu \mathrm{L}$ RNase-free water. The quality and concentration of the miRNA were detected using an ultraviolet spectrophotometer [the ratio of optical density $(\mathrm{OD})_{260} / \mathrm{OD}_{280}$ was 1.8-2.1].

\section{PC-3 transfection}

The sequence of the antisense oligonucleotide (ASO) of miRNA-181b was 5'-ACCCACCGACAGCAATGAATGTT-3', and that of the non-controlled ASO (ASO-NC) was 5'-CAGTACTTTTGTGTAGTACAA-3'. Transfection was performed with Lipofectamine 2000 (Invitrogen) according to manufacturer instructions. Cells were inoculated on 6-pore plates and cultured with $2 \mathrm{~mL}$ antibiotic-free medium in each pore on the day before transfection. The cells grew to $30-50 \%$ of the pore on the transfection day. The medium was replaced with $1.5 \mathrm{~mL}$ serum-free culture medium (GIBCO, Cat. No. 31985) $30 \mathrm{~min}$ before transfection. In the A solution, $240 \mathrm{~mL}$ Opti-MEM serum-free medium was added to $100 \mathrm{pmol}$ ASO-181b and oscillated gently. In the B solution, $240 \mu \mathrm{L}$ Opti-MEM serum-free culture medium was added to $10 \mu \mathrm{L}$ Lipofectamine 2000, oscillated, and allowed to stand at room temperature for $5 \mathrm{~min}$. The B solution was added to the A solution, oscillated, and then allowed to stand for 20 min. The mixed solution was transferred slowly and evenly onto the cells on the 6-pore plates for incubation at $37^{\circ} \mathrm{C}$. The cells were harvested after transfection for biological detection.

\section{RNA extraction}

Total RNA of the oligonucleotide- and plasmid-transfected PC-3 cells was extracted $72 \mathrm{~h}$ after transfection. The quality and concentration of the miRNA were detected using an ultraviolet spectrophotometer (the ratio of $\mathrm{OD}_{260} / \mathrm{OD}_{280}$ was 1.8-2.1). 


\section{Reverse transcriptase-polymerase chain reaction (RT-PCR)}

miRNA-181b expression in the 27 prostate cancer tissues, 30 normal prostate tissues, and transfected PC-3 cells were detected using RT-PCR. Total RNA ( $5 \mu \mathrm{g})$ was obtained for reverse transcription. The specific miRNA-181b reverse transcriptional primer was 5'-GTCGTATCCAGTGCAGGGTCCGAGGTGCACTGGATACGACCACCCACC-3' (Chen et al., 2005). The PCR primers were 5'-TGCGGAACATTCATTGCTGTC-3' (forward) and 5'-CCAGTGCAGGGTCCGAGGT-3' (reverse). Under amplification conditions, a denaturation step was carried out at $94^{\circ} \mathrm{C}$ for $4 \mathrm{~min}$, followed by 40 cycles at $94^{\circ} \mathrm{C}$ for 30 $\mathrm{s}, 50^{\circ} \mathrm{C}$ for $30 \mathrm{~s}$, and $72^{\circ} \mathrm{C}$ for $40 \mathrm{~s}$. After the reactions, the Ct value of the PCR curve was analyzed. U6 ribosomal RNA was used as the positive internal reference to correct cell copy number on the PCR template and eradicate error in the sample application amount, which was based on the following formula: $\Delta \mathrm{Ct}$ value of the target gene $=\mathrm{Ct}$ value of the target gene - U6 Ct value of the same sample. The procedure was repeated three times. The relative cyclic value of the target gene ( $\Delta \Delta \mathrm{Ct})$ was calculated based on the following formula: $\Delta \Delta \mathrm{Ct}$ value $=$ average $\Delta \mathrm{Ct}$ value of the treated group - average $\Delta \mathrm{Ct}$ of the control group. The $\Delta \Delta \mathrm{Ct}$ value was calculated using the $2^{-\Delta \Delta C t}$ method (Livak and Schmittgen, 2001).

\section{3-(4,5-Dimethylthiazol-2-yl)-2,5-diphenyltetrazolium bromide (MTT) assays}

PC-3 cell proliferation after transfection was detected using the MTT method. The PC-3 cell line was inoculated on a 96-pore plate, and $200 \mu \mathrm{L}$ antibiotic-free medium was added to each pore on the day before transfection. Cells grew to $30-50 \%$ of each pore on the transfection day. The medium was replaced with $50 \mu \mathrm{L}$ serum-free culture medium (GIBCO) $30 \mathrm{~min}$ before transfection. In the A solution, $24 \mathrm{~mL}$ Opti-MEM serum-free medium was added to $10 \mathrm{pmol}$ ASO- $181 \mathrm{~b}$ and oscillated gently. In the B solution, $24 \mu \mathrm{L}$ Opti-MEM serumfree culture medium was added to $0.25 \mu \mathrm{L}$ Lipofectamine 2000 (Invitrogen), oscillated, and allowed to stand at room temperature for $5 \mathrm{~min}$. The B solution was added to the A solution, oscillated, and allowed to stand for $20 \mathrm{~min}$. The mixed solution was transferred slowly and evenly into the cells of the 96-pore plate for incubation at $37^{\circ} \mathrm{C}$. The absorbance value at the wavelength of $490 \mathrm{~nm}$ was measured $4 \mathrm{~h}$ after transfection using a Quant spectrophotometer.

\section{Cell growth curve}

Transfected PC-3 cells were inoculated on 6-pore plates $24 \mathrm{~h}$ after transfection at a density of $1 \times 10^{5} /$ pore. Culture media were added. Cell numbers in three pores were counted in each group every day for 7 consecutive days. A cell growth curve was drawn with cell number on the $\mathrm{y}$-axis and time on the $\mathrm{x}$-axis.

\section{Flow cytometry}

Cell apoptosis was detected using flow cytometry with an ApoScreen AnnexinV Apoposis Kit (Shanghai Wuhao Trade Co., Ltd., China) $72 \mathrm{~h}$ after transfection. In the apoptotic analysis, FL1 (x-axis) presented the Annexin-V-R-PE fluorescent signal value for phosphatidylserine detection, whereas FL2 (y-axis) presented the 7-aminoactinomycin D (7- 
AAD) fluorescent signal value for DNA detection. Annexin-V-R-PE cannot stain normal cell membranes or 7-AAD. It stains apoptotic membranes positively but not 7-AAD. It stains both the membranes of necrotic cells or cells at the late stage of apoptosis and 7-AAD positively.

\section{Invasion detection}

In vitro invasion by PC-3 cells was detected using a Transwell chamber coated with Matrigel. Transfected PC-3 cells cultured in serum-free culture medium were placed in the upper layer of the Transwell chamber. RPMI 1640 culture medium containing 10\% fetal bovine serum was added to the lower layer. The cells were cultured for $24 \mathrm{~h}$. The chamber was removed. A mixed liquor was made by mixing methanol and glacial acetic acid in a $3: 1$ ratio for a 30-min fixation of the cells on the reverse side of the chamber. The cells were stained in crystal violet solution for $15 \mathrm{~min}$. Three visual fields were randomly chosen under the microscope, and photos were taken for the detection of the migrating cell number in the ASO group.

\section{Statistical analysis}

All data were analyzed using the SPSS 11.0 software. Analysis of variance was carried out. A level of $\mathrm{P}<0.05$ was considered to be statistically significant.

\section{RESULTS}

\section{miRNA-181 expression}

The miRNA-181 expression in tissues from 27 cases of prostate cancer and 30 cases of normal prostate was detected using RT-PCR. The results showed that miRNA-181b expression in the prostate cancer group was significantly higher than that in the normal group $(\mathrm{P}<$ 0.05; Figure 1).

\section{miRNA-181b expression after transfection}

Three groups were devised according to the various transfection methods. The ASO group comprised PC-3 cells transfected with antisense oligonucleotide. The ASO-NC group comprised cells transfected with non-controlled oligonucleotide. The control group comprised cells transfected with only liposomes. The RT-PCR results showed that miRNA-181b expression in the ASO group was significantly lower than that in any other group $(\mathrm{P}<0.05$; Figure 2).

\section{Cell proliferation}

Cell proliferation after transfection was detected using the MTT and cell growth curve methods. The results showed that PC-3 proliferation decreased with the decrease in miRNA$181 \mathrm{~b}$ expression (Figures 3 and 4 ). The result indicated that miRNA-181b promotes the proliferation of prostate cancer cells. 


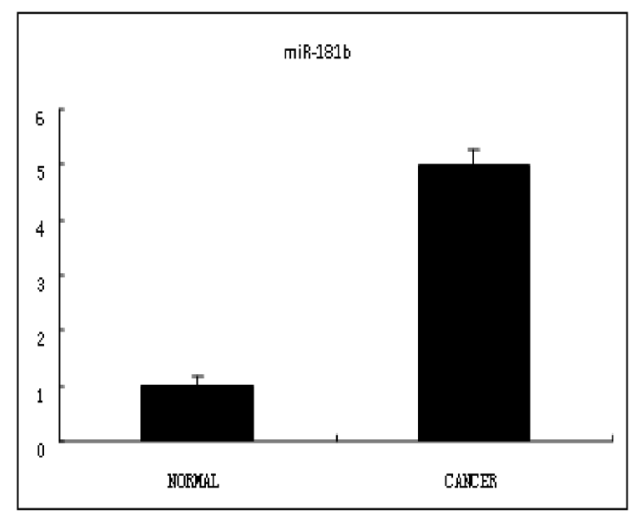

Figure 1. miRNA-181b expression in tissues from 27 cases of prostate cancer and 30 samples of normal prostate. A statistically significant difference was observed between groups $(\mathrm{P}<0.05)$.

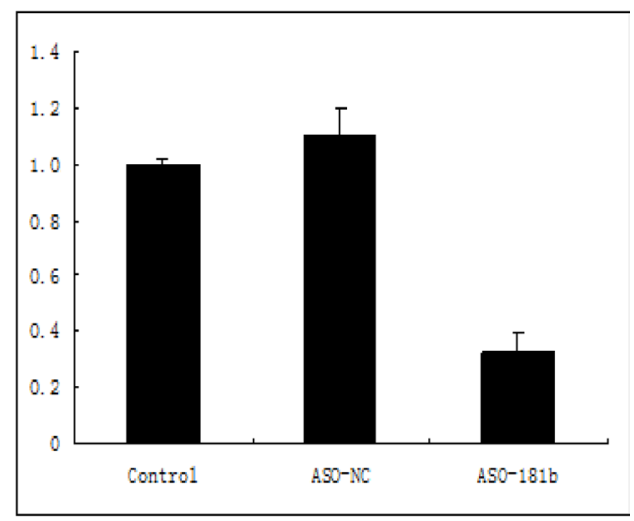

Figure 2. miR-181b expression in prostate cancer cells after transfection. A statistically significant difference was found in the antisense oligonucleotide (ASO) group compared with the control and non-controlled ASO (ASO-NC) groups $(\mathrm{P}<0.05)$.

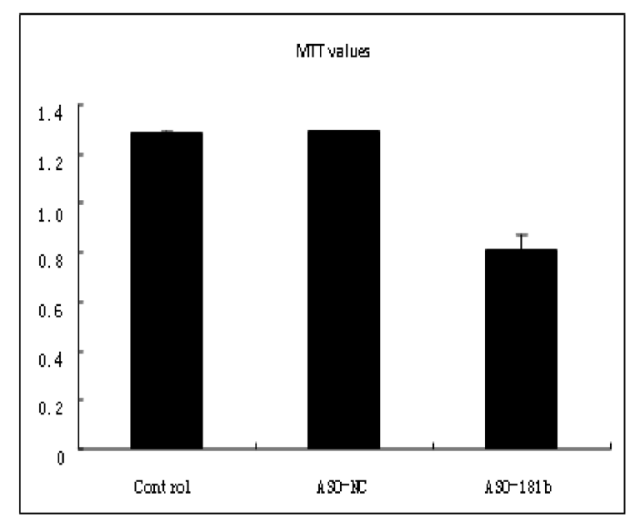

Figure 3. Influence of miR-181b expression on the proliferation activity of the PC-3 cells. MTT= 3-(4,5-dimethylthiazol-2-yl)-2,5-diphenyltetrazolium bromide. For other abbreviations, see legend to Figure 2. 

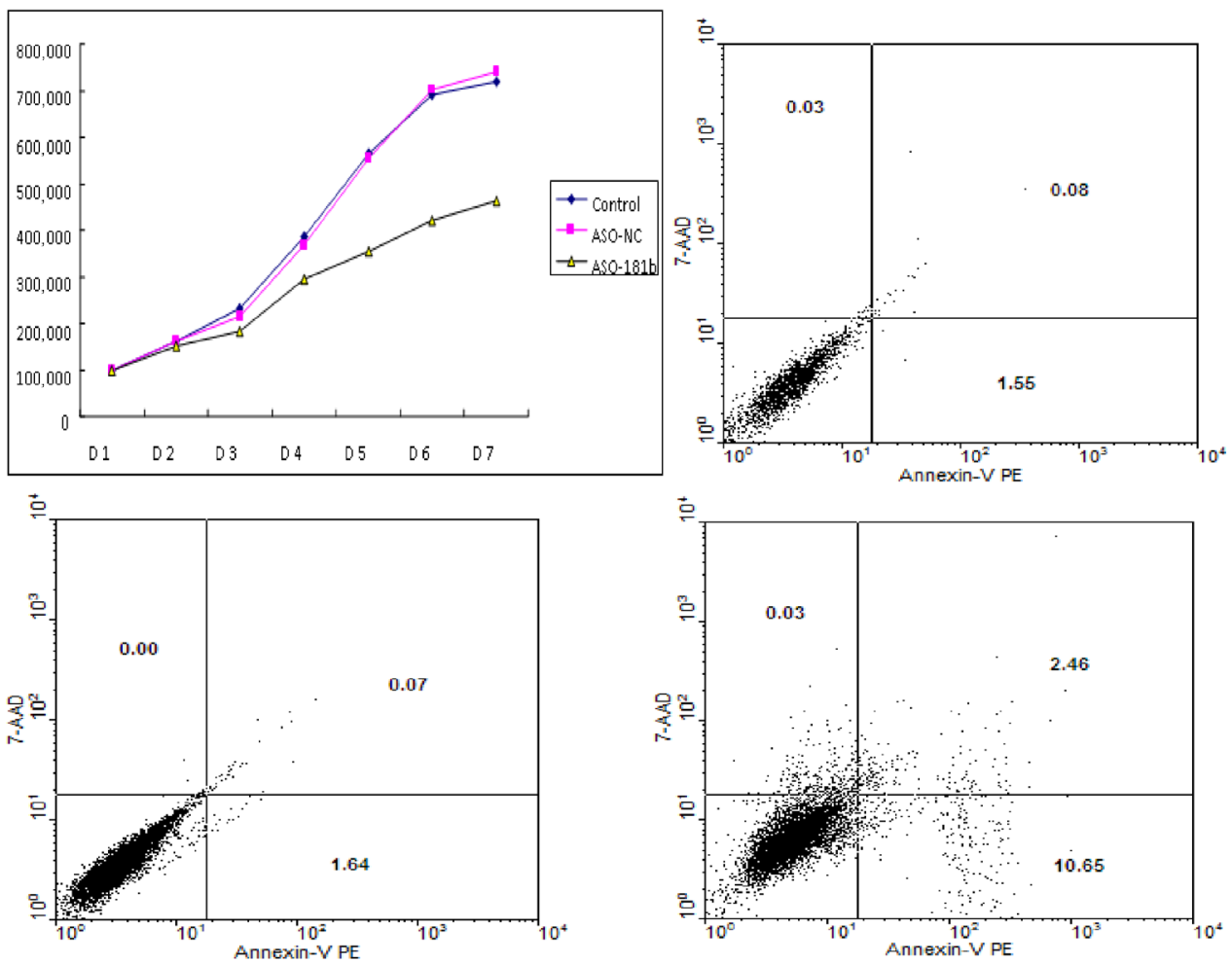

Figure 4. PC-3 cell growth curves after transfection. 7-AAD = 7-aminoactinomycin D. For other abbreviations, see legend to Figure 2.

\section{Apoptosis}

Cell apoptosis was detected $72 \mathrm{~h}$ after transfection using flow cytometry. The results showed that PC-3 apoptosis in the ASO group was obvious compared with that in the ASO-NC and control groups $(\mathrm{P}<0.05$; Figure 5). The result indicated that miRNA-181b has an inhibitory effect on the apoptosis of prostate cancer cells.

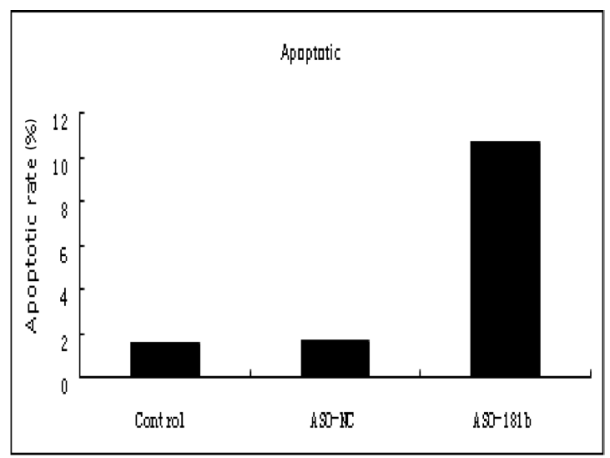

Figure 5. Cell apoptosis at $72 \mathrm{~h}$ after transfection using flow cytometry. For abbreviations, see legend to Figure 2. 


\section{Cell invasion}

PC-3 invasion was detected using the Transwell chamber method. The results showed that a decrease in miRNA-181b expression led to a decrease in PC-3 invasion (Figure 6), indicating that miRNA-181b overexpression promotes the invasion of prostate cancer cells.

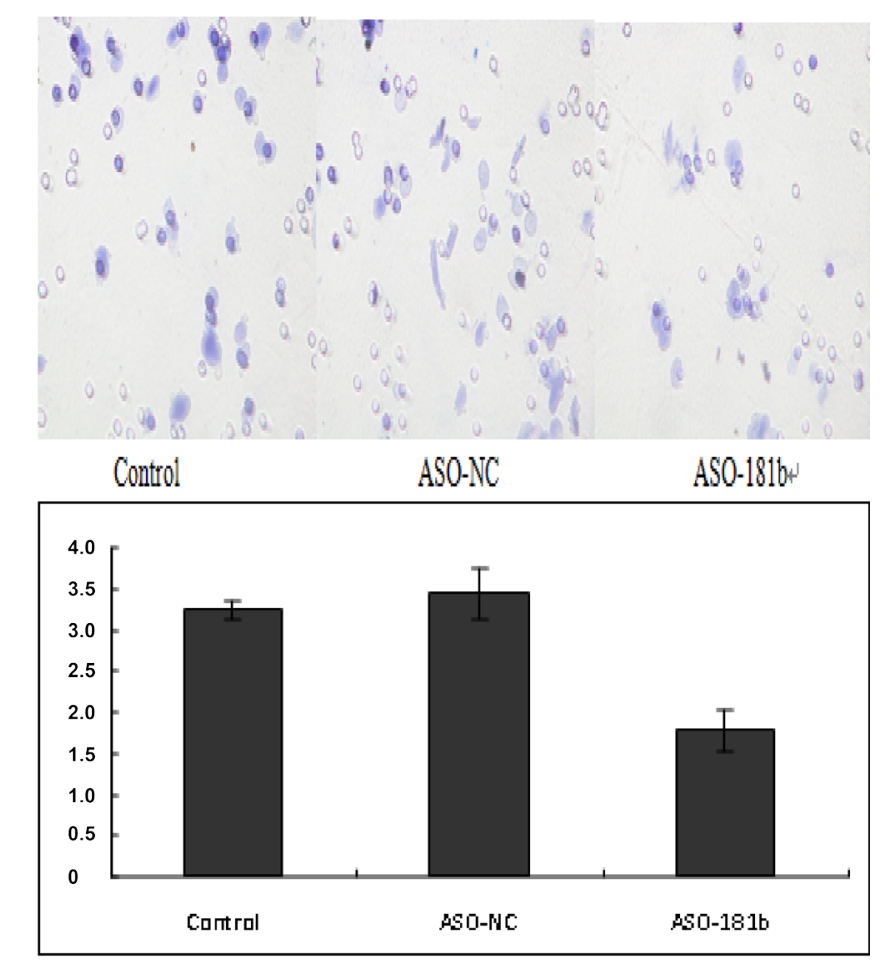

Figure 6. In vitro invasion of the PC-3 cells after transfection using the Transwell chamber analysis. For abbreviations, see legend to Figure 2 .

\section{DISCUSSION}

The genesis and development of prostate cancer is a complicated biological process involving the actions of numerous cytokines and interference genes. miRNA has a cancerinducing as well as a cancer-inhibiting function. Therefore, various levels of miRNA expression might play a role in the development of malignant diseases like prostate cancer. However, although some prostate cancer-associated genes have been discovered, their associated biological functions remain to be explored (Rajewsky, 2006).

Expression of miRNA-16, miRNA-125b, and miRNA-205 is down-regulated in 5-year non-recurrence cases, miRNA-96 expression is up-regulated in cases of 1-year recurrent malignant disease, and the expression of miRNA-221 and miRNA-222 is up-regulated in cases of long-term highly differentiated recurrent tumors (Schaefer et al., 2010). miRNA-21 expression is overexpressed in tumor tissues, and the associated targets are the bone morphogenetic protein receptor type II, the myristoylated alanine-rich protein kinase $\mathrm{C}$ substrate, and 
the programmed cell death 4 (Volinia et al., 2006). However, different results have also been reported (Spahn et al., 2010). Presumably, these disagreements may be caused by the varying methods used in these studies.

The miRNA-181 family is abnormally expressed in many tumors. Normally, the miRNA-181 family participates in the differentiation of thymocyte cells and regulates the ratio between B and T lymphocytes (Ambros and Chen, 2007). The miRNA-181 family is abnormally expressed in acute and chronic leukemia, and this expression is positively correlated with the morphological subtypes of acute leukemia (Debernardi et al., 2007). miRNA-181b regulates the proliferation of the leukemic cell line HL-60 through its target mixed lineage kinase 2 (Chen et al., 2009) and participates in the biological process of chronic leukemia by regulating T cell leukemia/lymphoma 1 expression (Pekarsky et al., 2006). The miRNA-181 family displays various levels of expression in tissues throughout the stages of human cerebral glioma, displaying a negative correlation with the stage of cerebral glioma (Shi et al., 2008). miRNA is abnormally expressed in colon cancer, and the abnormal expression is correlated with drug resistance (Nakajima et al., 2006; de Yébenes et al., 2008).

The current study showed that miRNA- $181 \mathrm{~b}$ is overexpressed in prostate cancer tissues, inhibiting miRNA-181b expression and promoting apoptosis, inhibiting proliferation, and weakening the invasive capability of PC-3 cells. These results indicate that miRNA-181b may be an oncogene in prostate cancer, participating in the processes of prostate cancer cell proliferation and invasion. However, its associated target genes remain to be explored further.

miRNA also serves as a cancer-inhibiting gene, as it has potential in tumor treatment. Prostate cancer-associated miRNA may be developed as a tumor treatment (Zhang and Farwell, 2008) based on the finding that this method can specifically inhibit cancer-inducing miRNA through its anti-miRNA action. The advantage of this method lies in its marked influence on multiple downstream factors of miRNA. Therefore, miRNA-mediated treatment may be more effective than traditional gene therapies are in the treatment of prostate cancer.

\section{REFERENCES}

Ambros V and Chen X (2007). The regulation of genes and genomes by small RNAs. Development 134: 1635-1641.

Berezikov E, Guryev V, van de Belt J, Wienholds E, et al. (2005). Phylogenetic shadowing and computational identification of human microRNA genes. Cell 120: 21-24.

Chen C, Ridzon DA, Broomer AJ, Zhou Z, et al. (2005). Real-time quantification of microRNAs by stem-loop RT-PCR. Nucleic Acids Res. 33: e179.

Chen H, Chen Q, Fang M and Mi Y (2009). Regulatory effect on the proliferation of the leukemic cell HL-60 by miRNA181b through MLK2 science in China. Life Sci. 39: 1034-1040.

de Yébenes VG, Belver L, Pisano DG, Gonzalez S, et al. (2008). miR-181b negatively regulates activation-induced cytidine deaminase in B cells. J. Exp. Med. 205: 2199-2206.

Debernardi S, Skoulakis S, Molloy G, Chaplin T, et al. (2007). MicroRNA miR-181a correlates with morphological subclass of acute myeloid leukaemia and the expression of its target genes in global genome-wide analysis. Leukemia 21: 912-916.

Gibson W, Green A, Bullard RS, Eaddy AC, et al. (2007). Inhibition of PAX2 expression results in alternate cell death pathways in prostate cancer cells differing in p53 status. Cancer Lett. 248: 251-261.

Jonler M and Pedersen KV (2007). Diagnosis, evaluation and follow-up of patients with prostatic cancer. Ugeskr. Laeger 169: 1889-1891.

Livak KJ and Schmittgen TD (2001). Analysis of relative gene expression data using real-time quantitative PCR and the 2(-Delta Delta C(T)) method. Methods 25: 402-408.

Marcucci G, Radmacher MD, Maharry K, Mrózek K, et al. (2008). MicroRNA expression in cytogenetically normal acute myeloid leukemia. N. Engl. J. Med. 358: 1919-1928. 
Meltzer PS (2005). Cancer genomics: small RNAs with big impacts. Nature 435: 745-746.

Nakajima G, Hayashi K, Xi Y, Kudo K, et al. (2006). Non-coding MicroRNAs hsa-let-7g and hsa-miR-181b are associated with chemoresponse to S-1 in colon cancer. Cancer Genomics Proteomics 3: 317-324.

Ozen M, Creighton CJ, Ozdemir M and Ittmann M (2008). Widespread deregulation of microRNA expression in human prostate cancer. Oncogene 27: 1788-1793.

Pekarsky Y, Santanam U, Cimmino A, Palamarchuk A, et al. (2006). Tcl1 expression in chronic lymphocytic leukemia is regulated by miR-29 and miR-181. Cancer Res. 66: 11590-11593.

Porkka KP, Pfeiffer MJ, Waltering KK, Vessella RL, et al. (2007). MicroRNA expression profiling in prostate cancer. Cancer Res. 67: 6130-6135.

Prueitt RL, Yi M, Hudson RS, Wallace TA, et al. (2008). Expression of microRNAs and protein-coding genes associated with perineural invasion in prostate cancer. Prostate 68: 1152-1164.

Rajewsky N (2006). microRNA target predictions in animals. Nat. Genet. 38 Suppl: S8-13.

Schaefer A, Jung M, Mollenkopf HJ, Wagner I, et al. (2010). Diagnostic and prognostic implications of microRNA profiling in prostate carcinoma. Int. J. Cancer 126: 1166-1176.

Shi L, Cheng Z, Zhang J, Li R, et al. (2008). hsa-mir-181a and hsa-mir-181b function as tumor suppressors in human glioma cells. Brain Res. 1236: 185-193.

Spahn M, Kneitz S, Scholz CJ, Stenger N, et al. (2010). Expression of microRNA-221 is progressively reduced in aggressive prostate cancer and metastasis and predicts clinical recurrence. Int. J. Cancer 127: 394-403.

Stark A, Brennecke J, Bushati N, Russell RB, et al. (2005). Animal MicroRNAs confer robustness to gene expression and have a significant impact on 3'UTR evolution. Cell 123: 1133-1146.

Volinia S, Calin GA, Liu CG, Ambs S, et al. (2006). A microRNA expression signature of human solid tumors defines cancer gene targets. Proc. Natl. Acad. Sci. U. S. A. 103: 2257-2261.

Xu L and Wang GM (2007). The progress and current situation in the management of moderate and far advanced prostate cancer. Int. J. Urol. Nephrol. 27: 773.

Zhang B and Farwell MA (2008). microRNAs: a new emerging class of players for disease diagnostics and gene therapy. J. Cell Mol. Med. 12: 3-21. 